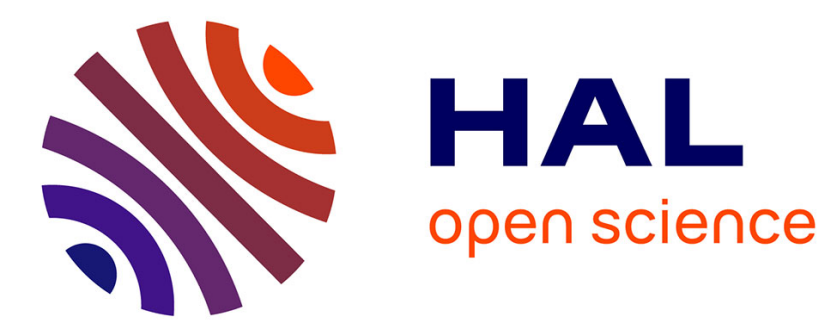

\title{
De Baltimore à Grigny, le traitement préférentiel des quartiers pauvres
}

Thomas Kirszbaum

\section{To cite this version:}

Thomas Kirszbaum. De Baltimore à Grigny, le traitement préférentiel des quartiers pauvres. Hommes \& migrations, 2003, pp.74-89. halshs-01100693

\section{HAL Id: halshs-01100693 \\ https://shs.hal.science/halshs-01100693}

Submitted on 6 Jan 2015

HAL is a multi-disciplinary open access archive for the deposit and dissemination of scientific research documents, whether they are published or not. The documents may come from teaching and research institutions in France or abroad, or from public or private research centers.
L'archive ouverte pluridisciplinaire HAL, est destinée au dépôt et à la diffusion de documents scientifiques de niveau recherche, publiés ou non, émanant des établissements d'enseignement et de recherche français ou étrangers, des laboratoires publics ou privés. 


\title{
De Baltimore à Grigny, le traitement préférentiel des quartiers pauvres
}

\author{
Solution participative expérimentale contre remède administratif national. Chacune exemplaire \\ dans son pays, les expériences de Baltimore (Maryland) et de Grigny (Essonne) mettent en évidence \\ deux politiques opposées de revitalisation des quartiers pauvres. Dans le cas américain, le programme \\ d'Empowerment zones cherche à impliquer la population dans le développement du site. \\ Dans le cas français, la politique des Grands projets de ville place le destin des habitants sous l'aile \\ protectrice des institutions publiques, qui décident entre elles et expliquent ensuite.
}

par Thomas Kirszbaum, doctorant en sociologie à l'université de Paris- $X$, chercheur au Centre d'étude, de documentation et d'observation sur les villes (Cedov)

1)- Cette enquête portait sur quatre sites : Chicago, Baltimore, Marseille et Grigny Viry-Chatillon. Thomas Kirszbaum, Le traitement préférentiel des quartiers pauvres. Les Grands projets de ville à la lumière de l'expérience américaine des Empowerment zones, rapport de recherche, PUCA-Fasild, décembre 2002.
Les minorités ethniques concentrées dans les quartiers pauvres ne sont pas seulement désavantagées en termes de revenus et d'égalité des chances, elles le sont aussi dans le domaine de la représentation politique. La persistance de la pauvreté dans ces lieux s'explique en partie par leur défaut d'influence sur la scène publique locale, là où se décide la répartition des ressources susceptibles d'influer sur leur bien-être économique et social. Comme la concentration de la pauvreté alimente l'exode des ménages les plus susceptibles de faire jouer leurs droits civiques (si tant est qu'ils en aient la jouissance), ceux qui restent sont plus dépendants encore des pouvoirs publics. Dans tous les pays confrontés à cette question, des politiques spécifiques ont été conçues pour aider ces territoires à sortir du cercle vicieux de la pauvreté et de la dépendance. Ces démarches reposent sur un principe analogue, consistant à injecter de manière temporaire des moyens exceptionnels sur des "quartiers cibles" afin que s'y enclenche un processus vertueux de développement leur permettant de relever à terme d'un traitement ordinaire. Mais la place des populations résidentes dans ces démarches de "traitement préférentiel" des quartiers est loin d'être identique d'un pays à l'autre. De ce point de vue, les modèles d'action étatsuniens et français se situent aux antipodes, comme nous avons pu l'observer lors d'une enquête portant sur les Empowerment zones (EZ) et les Grands projets de ville (GPV) ${ }^{(1)}$.

Le programme d'Empowerment zones a été adopté par le Congrès américain en 1993, à la demande de l'administration Clinton alors nouvellement parvenue aux affaires. C'est à vrai dire la seule politique d'envergure initiée par le pouvoir fédéral depuis les programmes de "guerre à la pauvreté" des années soixante. Circonscrites à un nombre réduit de quartiers et privées du soutien des républicains au Congrès, les Empowerment zones doivent être regardées comme une politique expérimentale, 
à la différence des Grands projets de ville, qui sont l'expression la plus récente (1999) d'une stratégie nationale s'affirmant comme toujours plus volontariste. Les GPV consistent eux aussi en une allocation ciblée des ressources publiques sur un nombre limité de sites. Dans les deux cas, la stratégie de revitalisation se veut globale, même si les Empowerment zones privilégient un traitement économique et les Grands projets de ville un traitement urbain.

Que révèle le rapprochement de ces politiques quant aux manières de répondre à l'enjeu de la marginalisation des quartiers pauvres sur la scène publique ? La démarche américaine cherche à élever ces quartiers et les personnes qui y vivent au rang de "sujets de l'action", capables de décider de leur avenir, ce qu'exprime la notion difficilement traduisible en français d'"empowerment". Lapproche française est inverse, car ce sont les pouvoirs publics qui prennent en charge le destin des quartiers et des individus. Les quartiers sont traités comme les "objets" d'une sollicitude institutionnelle au titre d'une "discrimination positive territoriale". On ne saurait donc imaginer réponses plus contrastées que ne le sont les démarches française et américaine face à un enjeu similaire, celui de la reconnaissance des "quartiers de minorités" sur la scène locale. Pour l'illustrer, les sites de Baltimore (Maryland) et de Grigny (Essonne) ont été retenus ${ }^{(2)}$. Ces choix ne sont pas fortuits, car ces deux expériences sont regardées dans leurs pays respectifs comme des réussites exemplaires.

\section{Des quartiers sujets de l'action: l'exemple de Baltimore}

Les quartiers sélectionnés pour recevoir le label "Empowerment zone" l'ont été à la suite d'une série d'appels à projets lancés par l'administration fédérale. Près de trois cents sites ont répondu au premier d'entre eux, en janvier 1994. Les critères de sélection décisifs étaient la qualité du projet présenté par les candidats et sa dimension participative. Le but de l'empowerment étant la prise en main de leur destinée par les bénéficiaires de ce programme de lutte contre la pauvreté, le pouvoir fédéral avait fait de leur participation effective à l'élaboration du projet une condition impérative d'éligibilité. Les dossiers de candidature devaient notamment préciser "les groupes et individus
2)- Le GPV de Grigny s'applique aussi à la commune de Viry-Châtillon. Par commodité et parce que le principal quartier concerné, celui de la Grande Borne, est principalement situé à Grigny, on parlera ici du "GPV de Grigny". 
ayant participé à la production du projet", expliquer "comment les participants ont été sélectionnés" et montrer "en quoi les participants pris comme un tout représentent la diversité raciale, culturelle et économique de la communauté"(3).

À Baltimore, l'idée de répondre à l'appel d'offres fédéral a d'abord été soutenue par des acteurs de la société civile - entrepreneurs, fondations, "organisations communautaires" ${ }^{(4)}$ - qui ont vite convaincu le maire de l'époque des chances de succès de la ville. Le maire a donc

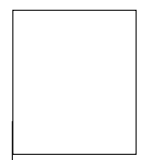

Il est courant d'entendre les acteurs locaux décrire l'empowerment comme une manière de s'éduquer.

Beaucoup se réfèrent à ce passage de la Bible où il est question d'apprendre à pêcher à un pauvre plutôt que de lui donner un poisson. installé une équipe chargée de superviser la fabrication du projet. Cette "Empowerment zone team" était composée dans un premier temps de fonctionnaires municipaux volontaires et issus aux deux-tiers de minorités ethniques (précision apportée par le dossier de candidature). Rapidement, des organisations communautaires ont été invitées à participer aux travaux. Deux mois plus tard, une première trame du projet fut présentée lors d'une réunion publique. Cinq cents personnes affluèrent, venues de tous les secteurs de la ville et notamment de ses nombreux quartiers défavorisés. Un appel était lancé aux bonnes volontés, pour qu'elles s'investissent dans l'écriture du projet. Cinq groupes de travail ont été constitués selon des principes suggérés par les participants.

3)- HUD (US department of Housing and

Urban development), Building communities: together, Empowerment zones \& Enterprise communities application guide, 1994.

4)- Aux USA, les organisations communautaires ("community-based organizations") recouvren un ensemble d'initiatives de la société civile dans les domaines urbain, économique, social, civique, etc. La ville de Baltimore (650 000 habitants) compte plusieurs centaine d'organisations de ce type.
Dès lors, le projet fut progressivement enrichi par un échange constant entre ces groupes et l'Empowerment zone team qui assurait la synthèse. Chaque étape devait être avalisée par une instance nommée par le maire, l'Advisory council. Un quart de ses membres représentait les quartiers pauvres de la ville ; les autres étaient issus des milieux d'affaires, d'institutions publiques ou d'organisations philanthropiques. L'Advisory council a donné un avis favorable au maire sur le projet final. Même s'il n'était pas forcément en accord avec l'intégralité de son contenu, le maire s'était engagé à respecter la volonté des acteurs de la société civile. Il a donc apposé sa signature.

Le dossier de candidature décrivait de façon très détaillée la nature des engagements pris par tous ceux qui s'étaient investis dans la préparation du projet. La compétition remportée, il fallait un cadre propice au maintien de la mobilisation. Comme suggéré par l'administration fédérale, une "structure de gouvernance" était mise sur pied. Ses membres étaient nommés par le maire, mais cette instance, appelée "Empowerment Baltimore management corporation" (EBMC), avait toute latitude pour piloter le projet. À l'image de ce qui s'était produit dans la phase précédente, EBMC devait représenter les diverses composantes de la société locale. Près de la moitié des trente sièges du conseil d'administration (board of directors) était attribuée à des représentants de la zone ; les autres étaient pourvus par les admi- 
nistrations de la ville et de l'État du Maryland par de riches hommes d'affaires, des responsables d'universités, de fondations privées ou d'organisations à but non-lucratif.

\section{Construire la communauté : ensemble}

Rapidement, l'élite du pouvoir local (économique et politique) a dominé le processus de décision, en occupant les présidences du conseil d'administration et des comités thématiques où se préparent les décisions avec l'aide des techniciens d'EBMC. Mais à l'inverse d'autres villes où le pouvoir de décider de l'allocation des fonds a été l'objet d'une âpre lutte, les représentants des quartiers pauvres ont pris leur parti de cette situation. Ils y ont vu une opportunité d'attirer des ressources nouvelles dans la zone et d'étendre leurs réseaux de relations à d'influentes institutions locales( ${ }^{(5)}$. Comme le dit le maire de l'époque, "l'Empowerment zone était une tentative de créer une passerelle entre les communautés pauvres et riches dans une ville qui reste terriblement divisée sur les plans social et racial". Outre le conseil d'administration d'Empowerment Baltimore, les activistes "communautaires" peuvent faire valoir leurs vues au sein de l'Advisory coun$c i l$, où ils disposent de la majorité des sièges. Chaque projet est examiné deux fois par cette instance consultative. "Le conseil d'administration ne prend aucune décision qui n'aurait pas l'agrément de l'Advisory council, assure sa présidente. Il nous donne la possibilité de faire entendre notre voix, ce qui n'est pas si fréquent lorsqu'on représente un quartier pauvre!"

L'esprit de coopération qui anime activistes communautaires et représentants du pouvoir local tranche avec l'expérience des années soixante. Les programmes urbains de la "guerre à la pauvreté", notamment le Community action program, étaient inspirés par le principe du "maximum de participation possible". Mais dans un climat marqué par une suspicion réciproque entre activistes et municipalités, les pouvoirs locaux n'avaient pas pris le relais de l'aide fédérale et les ghettos noirs demeuraient négligés sur la scène locale. L'expérience a légué toutefois un concept important, celui de "community organizing". Dans ces quartiers affectés par l'isolement et l'apathie, l'organizing vise à créer une force collective capable de défendre l'intérêt de la "communauté"(6). Aujourd'hui dominante, la tendance "réformiste" du développement communautaire a conservé cette idée que l'organisation de la communauté est une technique qui s'apprend par l'entremise de professionnels et peut se diffuser à travers des réseaux. L'organizing est pensé comme une étape dans la "construction d'une capacité de la communauté", capacité qui passe par la structuration d'organisations crédibles, capables de nouer des liens entre elles et ayant des partenaires extérieurs qui leur confient le développement de projets ${ }^{(7)}$. Le programme Empowerment zones recon-
5)- Sur les Empowerment zones comme vecteurs plus ou moins efficaces d'expansion des "opportunités civiques", cf. Marilyn Gittell (dir.), Empowerment zones: an opportunity missed. A six-city comparative study, The Howard Samuels state management and policy center, 2001.
6)- Le terme "communauté" est polysémique dans la langue anglaise. Il s'entend ici au sens du "quartier", ou simplement d'un groupe de personnes ayant un intérêt à agir ensemble.

7)- Le thème du "community capacity building" est au cœur de toutes les réflexions actuelles sur le développement communautaire aux ÉtatsUnis. Cf. par exemple Robert Chaskin et al., Building community capacity, Aldine de Gruyter, 2001. 
8)- Tel est le titre donné à une série de guides fédéraux destinés à accompagner les acteurs locaux dans leur démarche d'empowerment.

9)- Que l'on pourrait traduire par "tendre le bras vers". naissait cette nécessité, et se donnait pour devise de "construire la communauté : ensemble" (building communities: together) ${ }^{(8)}$.

À Baltimore, la "capacité d'action" des quartiers s'est construite, avec plus ou moins de succès, en appui sur six "Village centers" créés dans les frontières de la zone et regroupant chacun plusieurs quartiers. Ces organisations sont chargées d'assurer la participation continue des habitants, en même temps qu'ils leur délivrent des services liés au programme (insertion professionnelle, soutien aux familles, logement, etc.). Ils ont aussi la faculté de concevoir un "projet stratégique" sur tous les aspects de la vie des quartiers. À l'instar d'EBMC - la structure de gouvernance de l'Empowerment zone où ils envoient des représentants - les Village centers sont dotés d'un conseil d'administration et de comités thématiques où siègent les "stakeholders" (ceux qui ont un intérêt et quelque chose à investir), ainsi que d'une équipe technique. Certains membres siègent de droit aux conseils d'administration pour représenter telle ou telle organisation figurant dans les statuts du Village center (association d'habitants, regroupement d'entrepreneurs, organisation à but non lucratif, administration publique, hôpital, université...). D'autres sont élus directement par les habitants des quartiers concernés. Les réunions des Village centers sont ouvertes à tous et y participent des personnes désirant agir pour le bien de la communauté, car elles y trouvent un intérêt personnel ou une gratification symbolique, et surtout les représentants d'organisations chargées de développer les projets du quartier. Leur intérêt professionnel se confond avec celui de la communauté, ou du moins cherchent-ils à convaincre qu'il en va ainsi. Pour mieux s'assurer de leur représentativité, toute initiative de leur part réclame des lettres de soutien de la communauté, émanant de simples citoyens ou d'autres organisations.

En règle générale, seule une minorité d'habitants se mobilise. Pour sensibiliser les citoyens ordinaires, les Village centers recourent aux techniques classiques de l'“outreach"(9) : envoi de prospectus, invitation à des événements tels que le nettoyage des rues, distribution des comptes rendus des réunions ou de lettres d'information, etc. Les succès sont inégaux et les Village centers dans leur ensemble jugent la participation insuffisante.

\section{Apprendre à pêcher des fonds}

Les organisations de développement communautaire évoluent dans un environnement très concurrentiel, où l'on se bat pour le partage de ressources rares, publiques ou privées. Aussi, lorsque l'Empowerment zone fut annoncée à Baltimore, la perspective d'obtenir une part des cent millions de dollars a décuplé les appétits. La création des Village centers a montré la difficulté de bâtir des alliances entre organisations. Mais l'Empowerment zone les a incités à surmonter leurs conflits, car 


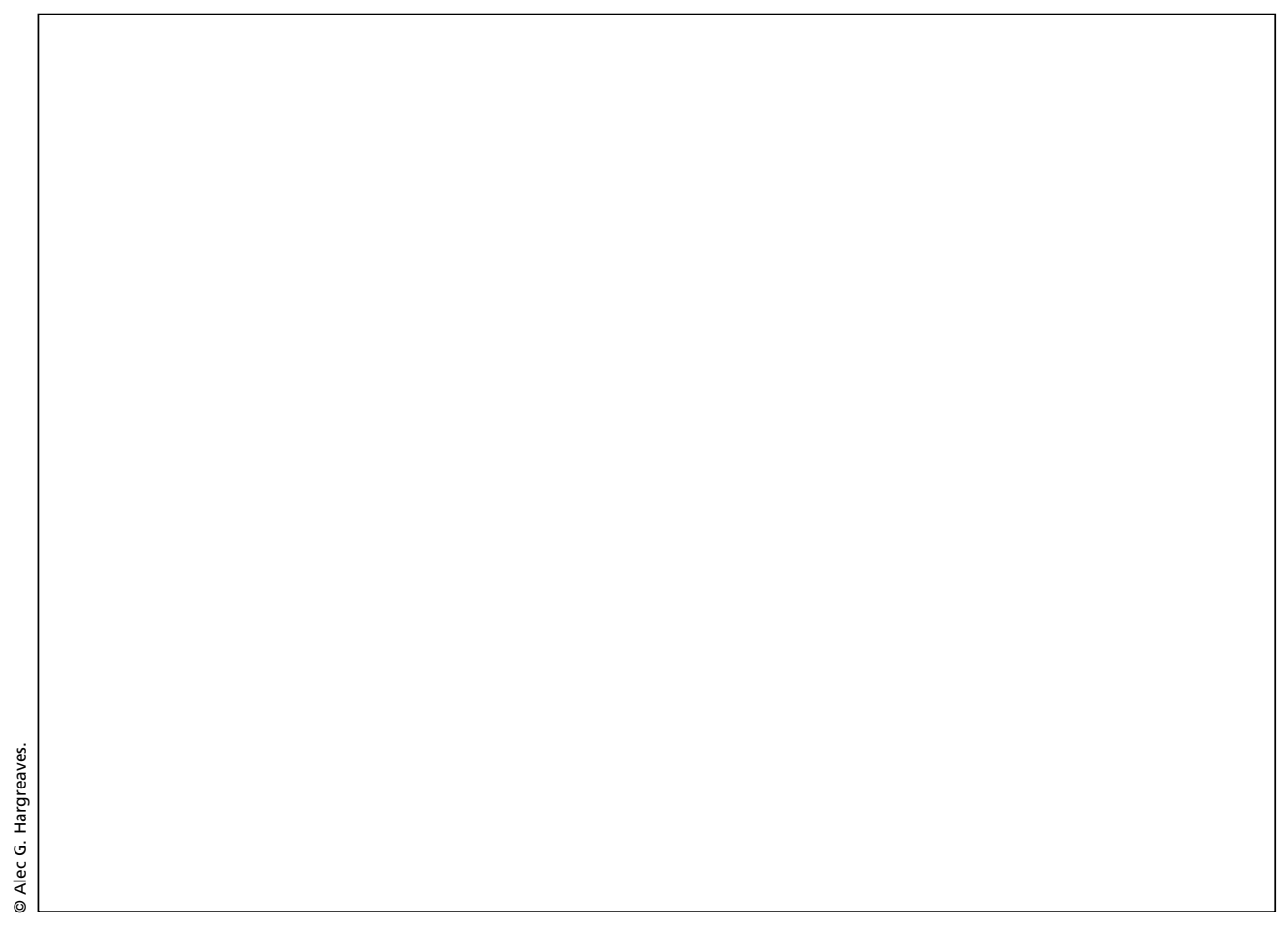

il fallait bien s'entendre pour recevoir des fonds et apparaître crédibles auprès de partenaires extérieurs. La plupart des Villages centers sont parvenus à établir un consensus, souvent grâce à l'abnégation de leaders reconnus pour faire passer leurs intérêts propres au second plan.

Il est courant d'entendre les acteurs locaux décrire l'empowerment L'accès au crédit pour tous reste un argument commercial pour cette compagnie de crédit qui vise ici la classe moyenne afro-américaine. comme une manière de s'éduquer. Beaucoup se réfèrent à ce passage de la Bible où il est question d'apprendre à pêcher à un pauvre plutôt que lui donner un poisson. Doter les quartiers d'une capacité d'action dépendra donc de "l'assistance technique" reçue de consultants, d'universités ou d'autres organisations communautaires. Elle porte sur tous les aspects de l'action communautaire : mobilisation des habitants, identification de leaders, réalisation du consensus, relations interraciales, management, connaissance des circuits institutionnels, etc. EBMC a mis à la disposition des Village centers une enveloppe de 1,2 millions de dollars pour rémunérer des consultants. S'ajoute l'aide en nature fournie par des universités, notamment par la School of social work de l'université du Maryland. Un aspect important de l'assistance technique porte sur la capacité des organisations à lever des fonds dans la perspective de durer. D'autant que l'Empowerment zone disparaîtra à la fin de l'année 2004 et que tous les Village centers ou presque sont ses créatures, et sont par conséquent dépendantes de ses subsides. Certains Village centers sont en bonne voie pour acquérir une autonomie financière grâce à des partenariats noués avec d'autres organismes 
publics, privés, philantropiques. La perspective qui s'ouvre pour les plus performants est celle de se transformer en Community development corporations, ce qui permet de conduire des activités lucratives (en matière de logement ou de développement économique principalement) dont les bénéfices sont ensuite recyclés dans la communauté.

\section{USA : transformer les politiques publiques par le bas}

Une idée forte du projet d'empowerment était de donner aux Village centers une capacité à attirer de nouvelles ressources en faveur des quartiers et à mettre en cohérence les ressources nouvelles ou existantes dans le cadre de leur "projet stratégique". Ils ont aussi la faculté d'élaborer un schéma directeur d'aménagement ("land use plan"), au terme d'une démarche participative. Ces démarches peuvent s'appliquer à tout domaine jugé important par la communauté du quartier et peuvent servir de référence dans les relations avec les partenaires. Un membre d'EBMC nous livre sa philosophie sur le sujet : "Le problème des quartiers pauvres ne sera pas réglé simplement parce que l'on mettra de nouveaux services à leur disposition. Le but est de donner aux communautés les moyens de s'organiser afin qu'elles puissent ensuite se retourner vers n'importe quel responsable institutionnel et lui dire : notre communauté a tel et tel besoin et voilà ce que nous proposons pour apporter un service de qualité."

Les Village centers bénéficient avec la municipalité de Baltimore d'un environnement favorable pour conduire ce type de démarches. Le cahier des charges fédéral des Empowerment zones demandait aux administrations locales de "se réinventer", pour faciliter la mise en œuvre du projet. À Baltimore, les innovations communautaires se diffusent avec une certaine aisance dans la culture administrative locale.

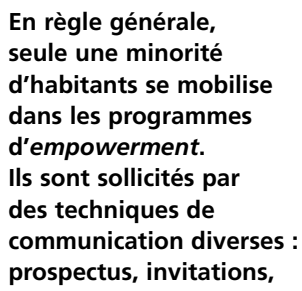


La ville est familière de cette idée que les quartiers pauvres ne sont pas seulement les destinataires des politiques publiques, mais doivent participer au processus de décision. Comme l'explique un fonctionnaire municipal, "les organisations communautaires constituent une source d'inspiration pour les institutions et ces dernières doivent descendre dans les quartiers pour rendre compte de ce qu'elles font". Cette formule de transformation "par le bas" des pratiques institutionnelles est loin d'être systématique, mais certains Village centers ont été des terreaux de l'innovation publique. Celui de Washington VillagePigtown, par exemple, mobilise régulièrement les habitants sur les enjeux liés à la sécurité, en même temps qu'il a su établir des relations de travail régulières avec les services de police et de justice.

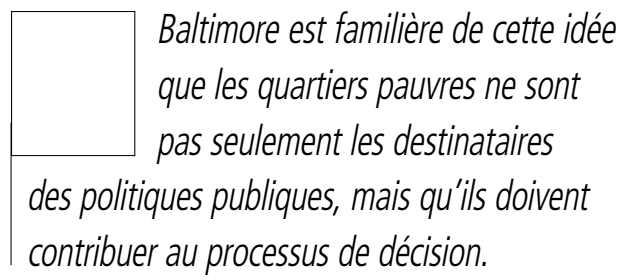
Autre exemple, celui de Harlem ParkLafayette Square, qui a mis en place un forum permettant aux habitants de discuter régulièrement des questions de sécurité, d'aménagement et de santé publique avec des représentants de la ville.

Les militants de la cause noire donnent volontiers un contenu politique à la notion d'empowerment (c'est le thème du "black political empowerment"). L'esprit du programme Empowerment zone est tout autre. Le dossier de candidature de la ville de Baltimore se réclamait certes de l'héritage de Saul Alinski, le père spirituel des techniques d'organisation des pauvres et de contestation des pouvoirs en place ${ }^{(10)}$. Le document adoptait un ton quelque peu irrévérencieux envers les autorités locales. Mais la limite du modèle est apparue une fois la structure de gouvernance (EBMC) lancée sur les rails. En son sein, les disparités de pouvoir se sont certes aplanies, chacun étant placé sur un pied d'égalité avec les autres. Mais les débats empreints de respect réciproque interdisent d'y porter des revendications dérangeantes pour l'establishment local. La conception civique du programme Empowerment zones est celle d'un pouvoir qui se révèle dans l'augmentation de la capacité des pauvres plutôt que dans l'affrontement avec les pouvoirs en place. Le pouvoir y est pensé comme un jeu à somme positive.

Un dilemme travaille cependant toutes les organisations de développement communautaire, qui voudraient concilier la mobilisation des habitants et le développement de projets. En pratique, la plupart de ces organisations dépensent une énergie considérable à faire du "networking" (de la "mise en réseau") avec des financeurs extérieurs, lesquels ont tendance à passer commande pour des projets ou des services plutôt que pour des actions visant à organiser les quartiers ${ }^{(11)}$. Cette synthèse difficile des dimensions mobilisatrice et instrumentale du développement communautaire a été observée à des degrés divers dans les

\author{
10)- Cf. son ouvrage \\ traduit en français, Manuel \\ de l'animateur social, \\ Seuil, 1978.
}

11)- Sur ce point, cf. Mark Joseph et Renae Ogletree, "Community organizing and comprehensive community initiatives", in R. Stone (dir.), Core issues in comprehensive communitybuilding initiatives, Chapin Hall Center for children, 1996. 
12)- Cf. Reid Cramer, Has the city development paradigm influence contemporary urban policy: evidence from the Empowerment zone program, University of Texas, non daté.

13)- Cf. par exemple Aspen Institute, Voices from the field. Learning from the early work of comprehensive community initiatives, 1997.

14)- Jean-Paul Delevoye (dir.), Cohésion sociale et territoires, rapport du Commissariat général au Plan, 1997.

15)- Jean-Pierre Sueur, Demain la ville, rapport présenté au ministre de l'Emploi et de la Solidarité 1998.

16)- Georges Cavallier (dir.), Nouvelles recommandations pour la négociation des contrats de ville de la nouvelle génération (2000-2006), rapport au ministre délégué à la Ville, 1999.
Empowerment zones ${ }^{(12)}$. Celle de Baltimore a permis de donner une voix aux habitants et de faire en sorte que l'expression des problèmes ne reste pas confinée dans l'enceinte des quartiers. Mais c'est là un aspect somme toute secondaire de l'activité des Village centers. S'ils peuvent s'autosaisir sur n'importe quel sujet, leur champ d'activité est borné par leurs obligations contractuelles vis-à-vis d'EBMC. Le plus gros de leurs financements dépend des résultats obtenus en matière d'accès à l'emploi. Les acteurs communautaires œuvrant en leur sein ont ainsi le sentiment que les aspects qualitatifs de leur travail (l'organisation de la communauté) ne sont pas assez valorisés. Une cause importante de hiérarchisation des activités résulte de la pression exercée par l'administration fédérale sur la production d'indicateurs quantitatifs montrant les progrès réalisés. Inhérent aux pratiques de développement communautaire, ce dilemme a été conceptualisé par la littérature spécialisée comme une tension entre les "outcomes" (le "produit final") et le "process" (le long processus d'organisation de la communauté) ${ }^{(13)}$.

\section{Des quartiers objets de sollicitude institutionnelle: l'exemple de Grigny}

Le programme des Grands projets de ville est intervenu au moment où de nombreuses voix pressaient l'État d'assumer sa fonction de "garant de la cohésion sociale et territoriale"(14) et les pouvoirs publics d'organiser "la ville de demain"(15). Dans le même temps, le concept de "renouvellement urbain" a été avancé pour inviter les institutions responsables du devenir des villes à travailler ensemble, afin de réduire la ségrégation sociale et ethnique dans les villes et de revaloriser les quartiers les plus lourdement pénalisés par leur image ${ }^{(16)}$. Les Grands projets de ville sont le principal instrument de cette stratégie de renouvellement urbain appliquée à des quartiers cibles. Quand le programme fut annoncé en 1999, le premier geste gouvernemental a consisté à rassembler autour de cette cause nationale ceux qui incarnaient au premier chef les pouvoirs publics : l'État local et les élus. La motivation de ces derniers à s'investir dans la démarche était même présentée comme une condition impérative pour espérer "gagner" un Grand projet de ville.

À Grigny et Viry-Châtillon, l'élaboration du projet a débuté par une grande messe inaugurale où le préfet, les maires et le président du Conseil général se sont assurés les uns les autres de leur détermination à s'engager dans une démarche devant préfigurer la formation d'un gouvernement territorial où ne manquerait aucun acteur institutionnel. La méthode ménageait une place résiduelle aux habitants et aux associations. À Grigny, des ateliers ont été mis sur pied à l'initiative de la ville et certaines associations ont été auditionnées à titres individuels. Mais leur implication était trop ponctuelle et les thèmes de 
réflexion trop cadrés (le social, l'éducation) pour qu'elles aient informé le projet de manière substantielle. La municipalité a fait la synthèse de cette consultation, puis a présenté aux associations l'avancement de la réflexion, sans les solliciter davantage jusqu'à la signature de la convention de Grand projet de ville.

Son élaboration s'est donc largement déroulée dans un entre-soi institutionnel. Le principe était simple : faire émerger le "point de vue" de chaque institution sur les problèmes territoriaux et la manière d'y porter remède, en assurer la synthèse et concevoir une stratégie à partir du croisement des thématiques et des territoires. Pour l'essentiel, les regards institutionnels ont convergé vers les mêmes "objets", des quartiers considérés comme lourdement handicapés. Sous la houlette d'un cabinet de consultants et du directeur du Grand projet de ville, les techni-

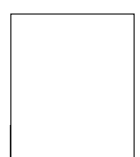

À Grigny, le discours des militants associatifs est une complainte sans fin sur le thème

du découragement des bonnes volontés

face au mur de la bureaucratie.

ciens des administrations locales ont été

les chevilles ouvrières de ce rapprochement interne à la sphère institutionnelle, sous le contrôle de ceux qu'ils désignent spontanément comme "les politiques", c'est-à-dire les élus et les hiérarchies administratives. La méthode grignoise a été saluée par les pouvoirs publics nationaux pour son caractère exemplaire, car elle a fait progresser l'unification du gouvernement territorial tout en fixant les devoirs de chaque institution de manière précise, et souvent chiffrée, dans une stratégie d'ensemble qui hiérarchisait les priorités ${ }^{(17)}$. La mise en évidence par le cabinet de consultants des écarts statistiques entre le territoire prioritaire et les moyennes départementales (sur l'emploi, l'éducation, la petite enfance, etc.) a été un moment décisif de la prise de conscience par les institutions d'un "devoir de faire plus et mieux" envers ce territoire très défavorisé. Les acteurs les plus impliqués dans le Grand projet de ville se sont ainsi reconnus dans l'idée d'une discrimination positive territoriale conçue comme un préalable obligé avant le retour à une gestion publique normalisée.

Il fallait un outil adapté pour maintenir les institutions mobilisées dans la durée. Un groupement d'intérêt public (Gip) a été créé à cette fin. Le Gip présente un double intérêt dans la perspective d'une unification du gouvernement local. Celui d'abord de mutualiser les différents apports financiers au sein d'un budget unique. Celui ensuite de la collégialité des décisions car l'assentiment de tous les membres est requis, poussant à l'unanimité. Le conseil d'administration du Gip rassemble les principaux décideurs publics locaux (préfet, maires, conseil général, directeurs des services de l'État, responsables d'organismes d'HLM, etc.) ${ }^{(18)}$. Ses décisions sont préparées par un comité de direction composé de techniciens de rang intermédiaire (sous-préfet à la ville, directeurs généraux des villes, responsables d'antenne des orga-

17)- Cf. L'éloge appuyé de la Cour des comptes dans son récent rapport sur la politique de la ville.
18)- Pour faciliter les transformations de la copropriété de Grigny II, l'association des copropriétaires a rejoint le Gip. 
Présentation, en 1995,

d'un projet

de réhabilitation de

la cité des Franc-Moisin

à Saint-Denis (93),

en présence d'Éric Raoult

ministre délégué à

la Ville et à I'Intégration.

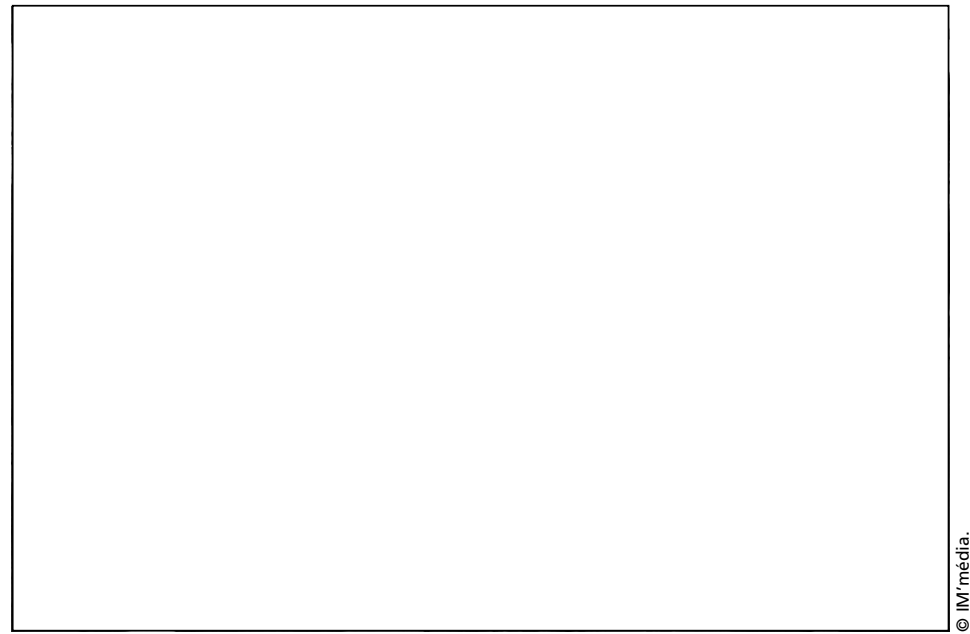

nismes HLM, chefs de service des administrations déconcentrées, etc.) qui joue un rôle charnière, en lien avec l'équipe technique du Gip, dans la construction de l'accord entre institutions.

\section{Agir au nom des minorités}

Les partenaires ont convenu que le Gip de Grigny-Viry n'établirait aucune relation directe avec les habitants ou leurs associations. Les initiatives à ce sujet seraient la prérogative exclusive des élus municipaux. En décembre 2002, soit deux ans après la signature du GPV, un comité consultatif des habitants a été installé à Grigny, après approbation du conseil municipal. Ce dernier a fixé les règles relatives à sa composition, distinguant plusieurs collèges à pourvoir selon un savant dosage. Celui des associations (quatorze sièges) a été pourvu à la suite d'un appel d'offre municipal. Une douzaine de candidats se sont déclarés, mais le bureau du conseil municipal en a écarté la moitié qu'il n'estimait pas assez représentatifs. D'autres associations ont donc été sollicitées de "gré à gré" pour prendre place au sein du comité consultatif. Un second collège comprend dix représentants élus par des "conseils de voisinage" dont l'accès est étroitement contrôlé par la municipalité. Le troisième est formé de dix élus municipaux désignés à la représentation proportionnelle des groupes politiques. Enfin, huit habitants ont été désignés pour représenter d'autres "forces vives" : quatre représentent la jeunesse (avec un souci de diversité des genres et des origines), deux les seniors et les deux derniers sont des commerçants. Comme son nom l'indique, ce comité aura un rôle consultatif. La municipalité sera libre de porter ses avis devant le comité du pilotage du Grand projet de ville.

Jusqu'à présent, le discours des militants associatifs grignois est une complainte sans fin sur le thème du découragement des bonnes 
volontés face au mur de la bureaucratie. Chaque association se sent isolée et les tentatives de rassemblement dans un collectif n'ont souvent d'autre finalité que de revendiquer de meilleures conditions pour le travail associatif. Les associations sont perçues et se perçoivent elles-mêmes comme des forces d'appoint des institutions, capables d'assurer des prestations "au plus près de l'intérêt des habitants", selon une expression de la convention de Grand projet de ville. Dans ce contexte, la fonction de représentation des associations est secondaire et leur représentativité toujours questionnée. Il est vrai que les bonnes volontés se recrutent souvent chez des personnes dont le profil social et ethnique n'évoque en rien celui de la majorité des habitants des quartiers. Ainsi la copropriété de Grigny II a un tissu de militants internes, formé pour l'essentiel de propriétaires résidents, assez âgés, en lutte contre la dévalorisation de leur bien et dans un rapport d'hostilité plus ou moins explicite envers la population d'origine immigrée responsable à leurs yeux de la perte de prestige de leur quartier.

Le profil des militants associatifs de la Grande Borne est d'une autre nature. Ils ont choisi de résider sur place, par souci éthique (nombreux sont des chrétiens de gauche), par conviction politique (beaucoup sont proches du parti communiste qui dirige la ville) ou pour des considérations professionnelles (les enseignants en poste dans le quartier sont le fer de lance du milieu associatif). Ces militants ne se défendent pas d'une vocation de "missionnaires" et se définissent comme les derniers vestiges d'une classe moyenne en voie de désertion. Tous affirment vouloir "passer le flambeau" à des jeunes du quartier plus proches de sa sociologie dominante. Mais c'est aussi à la sociologie qu'ils se réfèrent pour expliquer les barrières à leur engagement. "Ils se méfient de tout ce qui s'apparente à un moule, refusent l'autorité du collectif", analyse un militant. Plus vraisemblablement, les jeunes qui réussissent sont tentés de fuir le quartier ou sont incorporés dans des services municipaux de type "médiation de quartiers". Quant aux parents, ils se sentiraient "illégitimes" à s'exprimer pour des raisons statutaires (s'ils sont étrangers), linguistiques ou culturelles. Un élu met ainsi en avant quelques variables ethniques de la participation : "Selon les origines, certains sont plus ou moins prêts à s'investir. Nos Portugais [sic] ne sont pas très actifs. On aura plus de facilité chez les Asiatiques qui ont une certaine ambition. On ne voit en revanche aucune ambition chez les Antillais, contrairement aux Africains qui ont plus souvent acquis des formations secondaires ou supérieures dans leur pays d'origine." Se trouve évoquée aussi l'image dépréciée que leur renvoie la société locale, notamment "parce qu'ils n'ont pas envie de s'entendre dire que leurs enfants sont responsables des problèmes du quartier", selon un technicien municipal. À cela s'ajoutent les effets de l'instabilité résidentielle "qui ôte toute signification au sentiment d'appartenance à un quartier", d'après un tech- 
19)- Propos tenus par le ministre Claude Bartolone à Vaulx-en-Velin lors de la présentation du programme des acteurs de la politique de la ville, le 14 décembre 2000. Dans son discours,

le ministre demandait aussi que l'on s'interroge au plan national sur la mise en place du droit de vote

des étrangers aux élection locales... Avec le succès que l'on sait. nicien du Grand projet de ville. Dans ces conditions, rien de surprenant à ce "qu'ils restent entre eux, dans leurs associations communautaires", ajoute un militant associatif.

\section{France : transformer les politiques publiques par le haut}

Les Grands projets de ville n'ont pas dérogé à cette règle qui veut que le pouvoir national se livre à une surenchère rhétorique sur la participation des habitants chaque fois qu'une procédure nouvelle est lancée. Selon le ministre de l'époque, il s'agissait cette fois "de dépasser les discours nombreux en la matière, pour construire l'exercice d'une véritable démocratie locale"(19). Localement, l'État n'ira pas jusqu'à imposer de tels principes à ses partenaires des collectivités territoriales. Mais le discours national n'est lui-même pas dénué d'ambiguités. Au tournant des années quatre-vingt-dix, l'émergence du thème de la "citoyenneté" signalait un net glissement "républicain" du discours de la politique de la ville. Le diagnostic était simple : certains habitants de la ville sont moins citoyens que d'autres car les institutions républicaines les abandonnent.

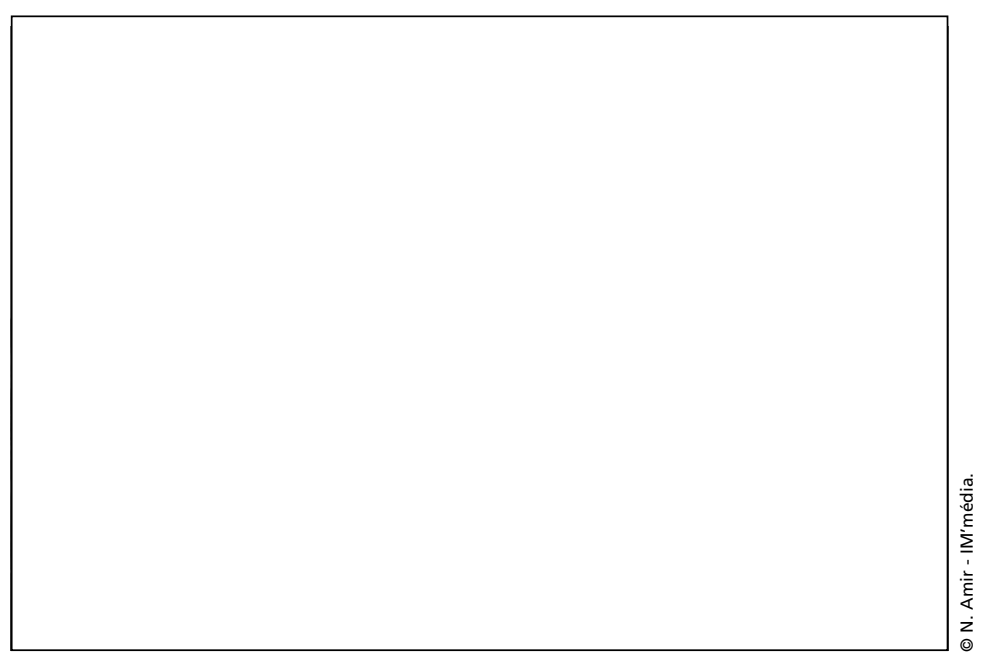

Lobjectif en découlait : promouvoir une citoyenneté réelle, par une mobilisation de l'action publique qui rétablisse le droit de ces habitants à être traités comme les autres. S'il était entendu, ce message portait en germe l'extinction de la nécessité même de la participation, le problème des habitants n'étant que le résultat d'une défaillance institutionnelle.

L'ambition civique des Grands projets de ville tient en une formule sans cesse réitérée : organiser le retour du droit commun (au sens des interventions publiques), pour mieux réinsérer les quartiers dans le droit commun (éviter la constitution de "ghettos" en marge de la 
République). S'ils veulent changer le mode d'allocation des ressources publiques, les acteurs des Grands projets de ville doivent avoir accès aux sommets du pouvoir local et convaincre les décideurs de s'investir dans le projet. La force du groupement d'intérêt public de Grigny vient en l'occurrence d'une bonne représentation des hiérarchies institutionnelles, et les premières évaluations montrent un réel effet levier sur les financements de droit commun. Cette expérience de Grigny peut être regardée comme une avancée dans la territorialisation des politiques publiques, mais aussi comme un modèle achevé (ou presque) du changement "par le haut" des pratiques publiques. La poli-

\begin{tabular}{|l|}
\hline $\begin{array}{l}\text { L'inspiration républicaine } \\
\text { des Grands projets de ville repose } \\
\text { sur l'unité supposée entre représentants }\end{array}$ \\
\hline $\begin{array}{l}\text { et représentés. Comme cette unité ne va } \\
\text { pas de soi, l'objectif est de réduire les décalages } \\
\text { par un travail de conviction des habitants. }\end{array}$
\end{tabular}
tique de la ville ne s'y trouve pas confinée dans les marges de l'action publique comme souvent ailleurs. Elle est directement prise en charge par les acteurs des politiques sectorielles représentés au plus haut niveau, tant du côté des techniciens que des décideurs. En vertu de cette approche, la transformation des pratiques institutionnelles doit se diffuser sur le territoire à partir d'injonctions venues d'en haut.

Les succès enregistrés par l'équipe de Grigny étaient encore improbables voici quelques années. Ces succès sont souvent expliqués par une réunion heureuse de personnes : un directeur de projet volontariste, capable de frapper aux bonnes portes et de donner le change aux décideurs publics, l'appui d'un bureau d'études faisant de la mobilisation du droit commun son cheval de bataille et armé d'une méthodologie laissant peu de répit aux techniciens des administrations, enfin le soutien sans faille d'un préfet pour qui la politique de la ville n'est pas la moindre des priorités. Mais la mobilisation institutionnelle reste facultative. Outre que la conjonction des personnes n'est pas éternelle (les deux premiers éléments du trio sont passés à d'autres missions), elle ne garantit pas de manière absolue la concrétisation des engagements institutionnels, car elle se heurte à une donnée structurelle : les principaux décideurs publics présents dans le Gip de Grigny représentent à la fois les intérêts du territoire et ceux de leur institution d'appartenance. Les décisions étant prises avec leur consentement, elles ne doivent pas entrer en contradiction avec les buts propres à leurs institutions. Le préfet donne un exemple : "La déontologie des administrations est contraire au principe de discrimination positive. Les administrations ont toujours la tentation d'égaliser le traitement des territoires pour minimiser le mécontentement des élus. Cela n'est pas compatible avec le constat que certains secteurs nécessitent des moyens, non en proportion de ce qui est demandé, mais de ce qui est nécessaire." 
20)- Conseil

interministériel des villes (CIV) du 30 juin 1998.

21)- Sur la notion de "pédagogie de la citoyenneté" et de "consentement" dans la politique de la ville, cf. Jacques Donzelot et al., Faire société aux États-Unis et en France. Deux réponses à la fracture urbaine, Seuil, 2003.

\section{Les limites de la sollicitude institutionnelle}

Les acteurs des Grands projets de ville ont conscience du scepticisme des habitants. La perte de confiance dans les institutions républicaines n'est pas propre à ces quartiers, mais les minorités ethniques qui y sont souvent majoritaires se reconnaissent moins que d'autres en elles. Les symptômes de cette désaffection (apathie politique, violences urbaines, sentiment d'injustice et d'abandon...) motivaient la "nouvelle ambition" de la politique de la ville ${ }^{(20)}$ : "refonder le pacte républicain" et convaincre les habitants de la détermination des pouvoirs publics à apporter des réponses décisives à leurs problèmes. Les Grands projets de ville devaient en être l'emblème. Plus important que leur propre mobilisation, il faut convaincre les habitants de la sollicitude de la société à leur égard et susciter leur adhésion au projet. Pédagogie et explication, tels sont les maîtres-mots de la relation aux habitants ${ }^{(21)}$. À Grigny, la convention stipule que "les membres du Gip s'engagent à mettre à disposition toute l'information nécessaire à la bonne compréhension de leurs projets, [et à] concerter les habitants et les usagers pour s'assurer de l'adéquation de la programmation à l'usage qu'en auront les bénéficiaires". De fait, la ville ne ménage pas ses efforts pour informer et expliquer, après avoir gardé le silence le temps que le projet prenne tournure. Une exposition a récemment été organisée pour visualiser le projet de transformation urbaine du quartier de la Grande Borne. "Les gens ont fini par comprendre et ont atteint le niveau de maturité suffisant pour accepter l'idée d'un projet urbain pour la Grande Borne", se félicite un technicien municipal.

Cette méthodologie du changement comporte une limite intrinsèque, celle de rendre les habitants entièrement tributaires des volontés institutionnelles et de la pertinence de leurs choix. Non seulement les habitants ne peuvent déterminer les priorités, mais ils n'ont guère de prise sur la réalisation des engagements. L'amélioration des performances publiques a un coût, mais la politique de la ville ne sait pas mesurer ni rendre compte de la réalité des efforts consentis et plus rarement encore démontrer les éventuelles inégalités de traitement subies par les habitants au regard d'autres territoires. "La discrimination positive est un principe souvent évoqué, mais il y a un écart fabuleux entre l'affichage et la réalité, observe le technicien d'un Grand projet de ville. Notre travail consiste à réduire cet écart entre le dire et le faire." Il en va en effet de la crédibilité de la parole publique dans une politique qui parle au nom de ses destinataires.

Empowerment zones et Grands projets de ville incarnent deux philosophies opposées de l'action préférentielle envers les "quartiers de minorités". La logique de l'empowerment se conçoit comme un mouvement qui part des habitants et d'organisations décidés à prendre en main leur destin. Mais les quartiers défavorisés ont besoin des res- 
sources externes pour se revitaliser. Une politique d'empowerment doit les aider à s'organiser et se doter des capacités nécessaires pour échanger avec l'extérieur, avec la "communauté élargie" ("community at large") disent les Américains. Ils parlent aussi d'une dynamique "inside-out"(22) et l'on pourrait dire de la démarche française qu'elle se déploie sur un mode inverse - "outside-in" -, partant des institutions publiques pour converger vers l'objet "quartier".

La conception de l'action collective à l'œuvre dans les deux pays repose sur des principes que l'on pourrait qualifier de "démocratique" dans un cas et de "républicain" dans l'autre. Démocratique aux ÉtatsUnis, car la logique du développement communautaire reconnaît toute sa place à la diversité des intérêts, ceux d'un quartier ou d'un ensemble de personnes regroupées dans un but commun. L'enjeu consiste à les transcender par la découverte d'un intérêt à agir ensemble. Comme dans toute entreprise démocratique, il y a péril lorsqu'une organisation perd de vue l'intérêt de ceux qu'elle dit servir. D'où l'importance du jugement des pairs et de la réputation qui s'acquiert ou se perd au sein des réseaux. L'inspiration républicaine des Grands projets de ville renvoie quant à elle à une logique unitaire de l'intérêt public. L'unité de vue entre représentants et représentés est l'axiome de cette politique. Et comme cette unité ne va pas toujours de soi, l'objectif est de réduire les décalages par un travail de conviction des habitants. L'écueil éventuel n'est pas lié comme aux États-Unis à la difficulté de déterminer un intérêt commun aux différents sujets de l'action, mais à la difficulté d'imposer à toutes les institutions la nécessité d'agir au bénéfice d'un objet - le quartier et ses habitants - et de résorber ensuite le décalage entre l'intention et la pratique. Ces deux conditions se réaliseraient-elles que la participation des habitants n'aurait plus de raison d'être.
22)- Cf. John Kretzmann et John McKnight, Building communities from the inside out: a path toward finding and mobilizing a community's assets, Center for urban affairs and policy research, Northwestern University, Chicago, 1993.

Jacques Donzelot et Marie-Christine Jaillet, "Fragmentation urbaine et zones défavorisées :

le risque de désolidarisation"

Jacques lon, "Injonction à participation et engagement associatif"

Francie Mégevand, "Participation des habitants : de l'expression des différences

à l'enjeu de cohésion sociale"

- Dossier La ville désintégrée ?, n 1217, janvier-février 1999

Abdelhafid Hammouche, "Quartiers sans travail, degré zéro de la sociabilité" Cyprien Avenel, "Quartiers défavorisés et ségrégation"

- Dossier Cités, diversités, disparités. À propos de quelques mécanismes de ségrégation, $n^{\circ} 1195$, février 1996

Laurence Gervais, "Chicago, chronique du ghetto"

- Dossier Australie, Canada, USA - Le multiculturel dans tous ses états, n 1174, mars 1994

France-USA : agir contre la discrimination - 1 - Philosophies et politiques 